\title{
CURRENT STATUS OF LIVER TRANSPLANTATION
}

Thomas E. Starzl, M.D., Ph.D.

Shunzaburo Iwatsuki, M.D.

Byers W. Shaw, Jr., M.D.

From the Department of Surgery, University of.Pittsburgh Health Center, University of Pittsburgh, Pittsburgh, Pennsylvania. .

Supported by research grants from the Veterans Administration; by project grant AM-29961 from the National Institutes of Health and by grant RR-00084 from the General Clinical Research Centers Program of the Division of Research Resources, National Institutes of Health. 
I would like to cover this morning the experience which we have had with liver transplantation and to try to indicate to you where I think developments in this field will be going. There has been some interesting administrative movement in the United States which may have relevance to the objectives of workers in Europe and the Middle East.

Liver transplantation has been considered to be an experimental undertaking and for that reason (and the consequent inability to fund these efforts) it has been difficult for centers other than the ones where I have worked to get started. However, the perception has grown in the last two or three years as results have improved with the introduction of cyclosporine that the procedure really is not experimental. There is in process in the United States an administrative chain of events known as a Consensus Development which will come to completion in June 1983 and which will almost certainly lead to a government decision that liver transplantation is in fact therapeutic.

When that time comes, we believe that a network of centers capable of providing liver transplantation will spring up in the United States almost overnight. We think that the number of centers will be 20 or 30 . There are already several active groups and there are in training several other teams. The steps in making liver transplantation a widespread service are following somewhat the same format as the development of renal transplantation about 20 years ago in our country. 
THE DIMENSIONS OF THE PROBLEM

Data about the need for hepatic transplantation is being accumulated. It has become obvious that the numbers of potential candidates are far higher than anyone had realized. Until recently no one had taken a good look at this question, perhaps because it was perceived to be a non-issue, but my estimate is that at an equilibrium level the number of liver transplantations in the Unites States will be in the 5,000 per year range. Many of you know that this is about the number of renal transplantations in the United States per year. In our country, liver disease is the fourth most common cause of death.

Although a significant proportion of these deaths are alcohol related, a surprising percent are free of the alcohol taint. Moreover, there is no reason to arbitrarily exclude patients with alcoholic cirrhosis from treatment. A number of years ago it was estimated that there might be as many as 5,000 potential candidates for liver transplantation in the British Isles and 20,000 in the United States (1). Although these figures are probably too high, it is clear that liver transplantation will be a common form of transplantation with numbers that may be competitive with the kidney. Realization of the objective of making liver transplantation a common service will depend upon the progress which $I$ will be talking about today .

\section{HEPATIC REJECTION AND ITS PREVENTION}

Rejection of liver grafts is not different in principle than that which is seen with other transplanted organs. In untreated 
dogs after 4 or 5 days, the typical findings include massive infiltration of mononuclear cells which are heavily concentrated around the portal tracts and central veins. Parenchymal necrosis and interstitial edema are other classical findings $(1,2)$.

Almost two decades ago, the feasibility of hepatic transplantation in patients with end stage liver disesase was established with the chronic survival of many dogs whose livers were replaced with grafts from non-related mongrel donors under treatment with azathioprine $(1,2)$. More than a dozen of the animals had therapy discontinued after about 4 months. Rejection did not supervene (1) and one of these dogs lived for more than a decade. The observation that therapy could often be stopped in these dogs as well as later work in Paris, Bristol, Cambridge, and Denver with pigs suggested that the liver could be at the "easy" end of the scale in terms of controlling rejection. That advantage, if it does exist, can be, easily overstated because rejection occurs frequently after human liver transplantation in spite of all efforts to prevent this process.

The first extended survivals after human liver transplantation were obtained in the summer of 1967 (1). In January 1970 a child was treated who became the human counterpart of the canine experiment in that her survival first equaled and then superceded that of the famous dog. She is now $13^{1 / 2}$ years post transplantation. I should say in passing that George Abouna was a member of the team that took care of this little girl. her treatment was with azathioprine, prednisone, and ALG. 


\section{INDICATIONS FOR LIVER TRANSPLANTATION}

The indications for liver transplantation in 237 consecutive cases are shown in Table 1 . The heterogenous nature of the diseases that can be treated is obvious. Chronic aggressive hepatitis, or more accurately non-alcoholic cirrhosis, was a leading indication, cutting across all age barriers. Biliary atresia was very common in pediatric patients.

With all the indications in Table 1 , we have tried to formulate some idea about the technical difficulties that the surgeons can anticipate at the time of transplantation, the average degree of metabolic abnormalities that can be expected from the original hepatic disease, the difficulties (if any) in deciding the propriety of candidacy, and finally the question of recurrence of the disease which had destroyed the native liver.

In Table 2 is a brief declaration of these issues in patients with biliary atresia. With this disorder, the decision about candidacy is a very easy one. Willis Potts, the great pediatric surgeon in Chicago, once wrote that biliary atresia had provided the blackest chapter in the history of pediatric surgery. His remarks were apt, since at that time nothing could be done for these children who required a major social, economic and medical input, but from whom nothing could be expected. The victims of biliary atresia became pariahs as they entered the downhill slope of their life with the development of numerous secondary complications. The breakdown of parent-offspring relationships in which the children became objects of despair and sometimes even hatred has been documented by social scientists. 
Thus the decision to operate on a child with biliary atresia is an easy one.

All patients with biliary atresia have had previous surgery, and this is an adverse factor, but not such a serious one as in adults. The most important technical problems with children with biliary atresia have come from associated anomalies, such as hypoplasia of the portal vein. The metabolic abnormalities at the time of transplantation have ranged from minor to very severe, but usually they have not been unreasonable. In the past many of these children have died from the complications of biliary atresia while still possessing good synthetic functions of their liver.

The same questions shown in Table 2 can be asked about all the major diseases for which transplantation might be indicated. Prospective recipients with non-alcoholic cirrhosis have been murderously difficult from a technical point of view and especially so when previous operations have been carried out within the abdomen. The degree of metabolic decay has been unusually severe in such patients. Finally patients with chronic active hepatitis who are $B$ virus carriers have had a marked tendency to recapitulate in their graft the same disease as destroyed their native liver. It now appears that the recurrence rate will be in the $80 \%$ range. Clearly, the full exploitation of liver replacement in such recipients will depend upon better ways of preventing a recurrence of the hepatitis. The use of hyperimune specific globulin, or the use of interferon are two possibilities. 
The inborn errors of metabolism (Table 1) have provided for surgical scientists and for biochemists a unique opportunity. It was discovered almost two decades ago that a new liver provided new protein phenotypes for the recipient and that these donor specific phenotypes were retained for the life time of the graft. This principle of donor specificity' of the liver homograft implies that hepatic based inborn errors of metabolism are curable by liver transplantation, and this implication has been proved on a number of occasions. The inborn errors shown in Table 1 (footnote) have all been metabolically "cured" with the possible exception of the sea-blue histiocyte syndrome. With congenital tyrosinemia and the glycogen storage diseases, there is a known and highly specific enzyme deficit in the livers; it has been shown that these enzymes when brought to the recipient with the new liver are thereafter present as long as the liver is viable. In other diseases such as Wilson's disease and alpha-1antitrypsin deficiency in which the exact enzyme deficit (if any) is not known, these disorders are also cured in spite of this ignorance about the basic pathogenesis.

Some of the most difficult decisions about candidacy have been in patients with hepatic malignancies $(1,3)$. The reason is that the incidence of recurrence in patients whose livers were replaced because primary tumors could not be removed with subtotal resection have exhibited a very high incidence of recurrence, more than $80 \%$ in our initial experience. In recent years, the incidence of recurrence has been lower (3) but this may only have reflected a better case selection. Many of the 
recipients with hepatic malignancies treated in the last several years have had the so called fibrolamellar hepatomas which are late to metastasize, or the hepatomas which occur as incidental findings in livers destroyed by other diseases such as tyrosinemia, alpha-1-antitrypsin deficiency, and the seablue histiocyte syndrome. The role of liver replacement in the treatment of patients with otherwise nonresectable hepatic tumors has yet to be fully defined, but it is certain that the minority of patients would qualify.

A number of diagnoses which are not well represented in Table 1 are certain to be more common in candidates for liver transplantation in the future. The most important of these diseases will be primary biliary cirrhosis and sclerosing cholangitis. The technical ease with which liver replacement can be carried out in primary biliary cirrhosis is remarkable. Patients with this disorder have normal or sometimes larger than normal livers, with relatively modest portal hypertension, and with a disease evolution that is slow enough so that therapies can be carried out early before terminal deterioration of health. The diagnosis of sclerosing cholangitis has a less favorable set of circumstances on the average since so many patients with this disease have either had total colectomy for the underlying disease of ulcerative colitis or else efforts at biliary tract reconstruction.

\section{TECHNICAL ASPECTS OF TRANSPLANTATION}

The preservation methods which can be used today are limited, but good enough to permit the harvesting of organs in 
various parts of the United States with transfer of the hepatic grafts for thousands of miles. In Europe, similar networks have been set up leading to English, German and Dutch centers.

The techniques of the actual transplantation have been so well described that there is little point in discussing these here $(1,3)$. A recent development which is going to play a major role in permitting transplantation to be used on a broad scale has been the use of veno-venous pump driven nonheparin bypasses. With such bypasses, the blood from the occluded vena caval system as well as from the portal vein can be shunted around the upper abdomen during the period of obligatory venous obstruction and channeled into the axillary vein or some other vein of the upper half of the body. The physiologic stress imposed in the past during the so called anhepatic phase has been largely eliminated by this method which has been used for the last 20 or 25 adults. It is probable that the perfection of these nonheparin bypasses has been the most important technical development in almost 2 decades.

In the early days of liver transplantation complications of biliary tract reconstruction were both frequent and lethal. These have been virtually eliminated in recent years. In our center the preferred form of biliary reconstruction is with duct to duct anastomosis over a T-tube. Obviously, this option does not exist for many patients (and by definition never with biliary atresia) but a very satisfactory alternative procedure is choledocojejunostomy using a Roux limb. 


\section{SURVIVAL}

With Conventional Immunosuppression (1963-1979)

From 1963 to the end of 1979170 consecutive patients were treated, an average case load of less than a dozen a year. Fifty-six (32.9\%) of the recipients lived for at least one year and $32(18.8 \%)$ are still alive with followups of $3 \frac{1}{2}$ to $13 \frac{1}{2}$ years. Six of the residual group are more than 10 years postoperative and 26 are more than 5 years. Only one patient who lived for as long as 5 years has subsequently died.

The predominant mortality was in the first 3 postoperative months and was due mainly to technical surgical accidents, acceptance of some recipients with hopelessly advanced disease, the use of damaged liver grafts, the inability to control rejection, and a variety of infections. The majority of the deaths subsequent to this time were due to chronic rejection.

With Cyclosporine-Steroid Therapy (1980-1981)

Fourteen patients were treated in 1980 and 26 in 1981; followups of 18 to 38 months are available for those still living. The one year survival was $28 / 40$ (70\%). Three of the one year survivors died in the 13,16 , and 20 th months of recurrent cholangiocarcinoma, recurring Budd-Chiari syndrome and chronic rejection (with an unsuccessful attempt at retransplantation) respectively.

The Breakout Year of 1982

In 1982,2 important events occurred which demonstrated that liver transplantation could be exploited in many centers. For one thing, a large number of transplantations was carried out, 80 
in all, an unprecedented number since the highest total in any previous year was only 30 . In addition, fellows and residents began to do the operations, and contributed almost half of the cases. During 1982, half of the recipients have survived with followups of 5 to 15 months. The losses still occurred perioperatively.

The trials with the non-heparin bypass during the actual transplantation were begun late in our experience and the results in 1983 will have to be assessed before a final decision about the influence of this achievement will be forthcoming.

In the meanwhile the movement of liver transplantation from an experimental to a service undertaking can be illustrated by studying the first 67 consecutive cases in what have been known as the cyclosporine era. The comparison. of the results using this drug with those in all previous experience is shown in Table 1. Results have been about twice as good as in the past (Table 1).

\section{Prospect for the Future}

The revitalization of interest in hepatic transplantation has dated from the better immunosuppression that became available $3^{1 / 2}$ years ago. Since that time the number of successful liver replacements has sharply increased.

Forty-two patients have been followed for at least a year after liver transplantation under cyclosporine-steroid therapy. The mystique surrounding liver transplantation has largely been dispelled during this time. Since 1980 other units in the United States and in other countries have been able to mount new and 
effective programs. Thus liver transplantation has been developed to the point of a service operation, the exploitation of which depends on the establishment of multiple regional centers. The increased use of this procedure will permit the delivery of optimal health care to victims of end stage liver disease. 


\section{REFERENCES}

1. Starzl TE (with the assistance of Putnam, CW): Experience in hepatic transplantation. Philadelphia, WB Saunders Co., 1969 .

2. Starzl TE, Marchioro TL, Porter KA, Taylor PD Faris TD, Herrmann TJ, Hlad CJ, Waddell WR: Factors determining short-and long- term survival after orthotopic liver homotransplantation in the dog. Surgery (58) 131-155, 1965 .

3. Starzl TE, Iwatsuki S, Van Thiel DH, Gartner JC, Zitelli BJ, Malatack JJ, Schade RR, Shaw BW Jr, Hakala TR, Rosenthal JT, Porter KA: Evolution of liver transplantation. Hepatology (2) 614-636, 1982. 


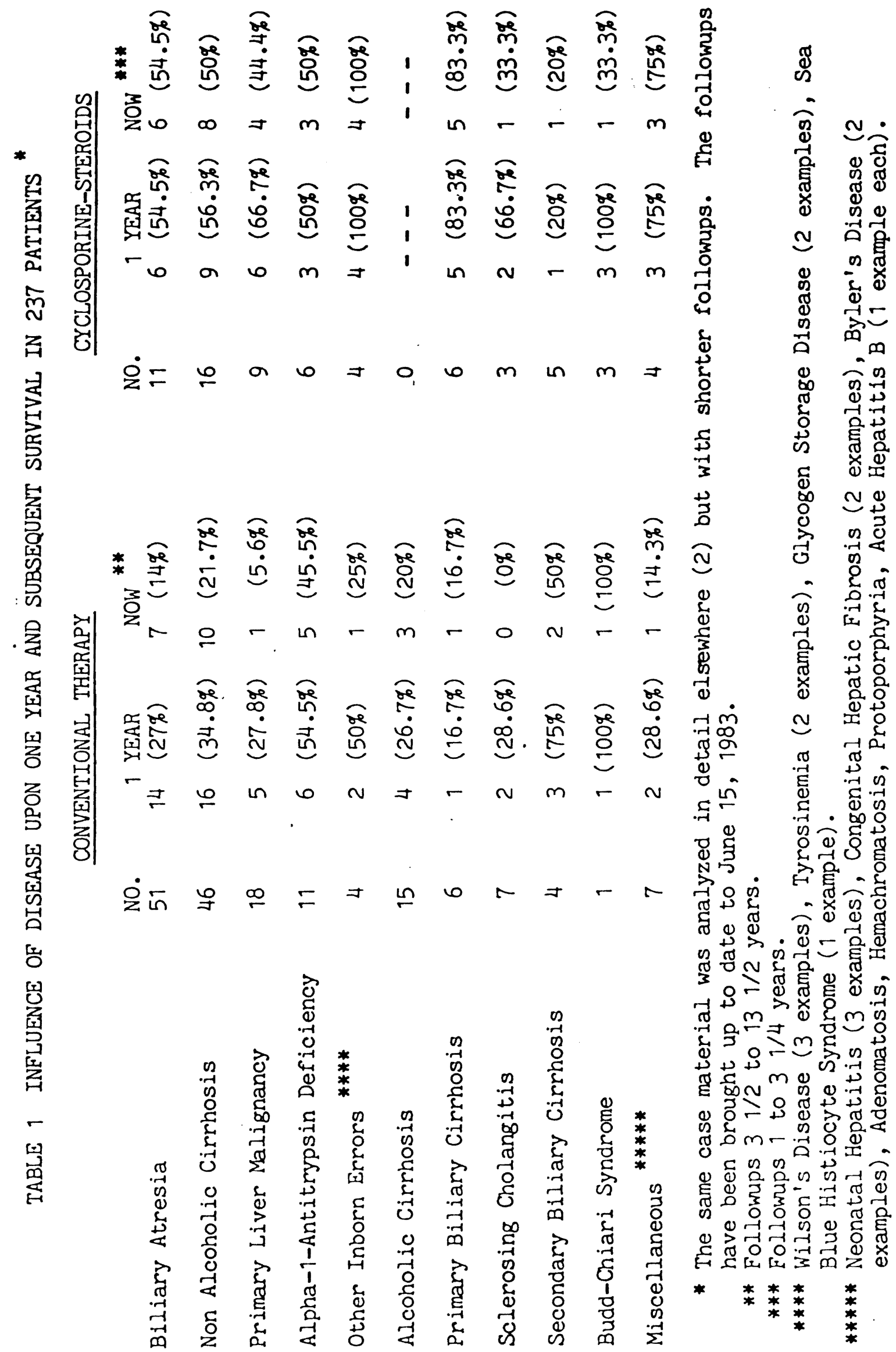




\section{Table 2. Factors of candidacy from experience with 237 consecutive patients. Biliary Atresia, 62 of the 237 cases}

Decision of Candidacy - - . - . - - Easy

Previous Surgery _... $\ldots \ldots \ldots$. $\ldots$.

Metabolic Abnormalities _ . . . . . . . - Moderate

Technical Difficulties _.......... Moderate ${ }^{*}$

Disease Recurrence _ . . . . . . . None

* Unless there are anomalies 